\title{
A reliable and affordable 3D tumor spheroid model for natural product drug discovery: a case study of curcumin
}

Loh Teng Hern Tan ${ }^{1,2,3 *}$, Liang Ee Low ${ }^{4,5}$, Siah Ying Tang ${ }^{4,5}$, Wei Hsum Yap ${ }^{6}$, Lay Hong Chuah ${ }^{7}$, Chim Kei Chan ${ }^{8}$, Learn Han Lee ${ }^{1,2,3,9,10}$, Bey Hing Goh ${ }^{1,2,7,9,10 *}$

${ }^{1}$ Biofunctional Molecule Exploratory Research Group, School of Pharmacy, Monash University Malaysia, 47500 Bandar Sunway, Selangor Darul Ehsan, Malaysia.

${ }^{2}$ Novel Bactria and Drug Discovery Research Group, Microbiome and Bioresource Research Strength Jeffrey Cheah School of Medicine and Health Sciences, Monash University, Bandar Sunway, Malaysia.

${ }^{3}$ Jeffrey Cheah School of Medicine and Health Sciences, Monash University Malaysia, 47500 Bandar Sunway, Selangor Darul Ehsan, Malaysia.

${ }^{4}$ Chemical Engineering Discipline, School of Engineering, Monash University Malaysia, Bandar Sunway, Malaysia

${ }^{5}$ Advanced Engineering Platform, Monash University Malaysia, Bandar Sunway, Malaysia

${ }^{6}$ School of Biosciences, Taylor's University, Subang Jaya, Selangor, Malaysia

${ }^{7}$ School of Pharmacy, Monash University Malaysia, Bandar Sunway, 47500, Subang Jaya, Selangor, Malaysia

${ }^{8}$ de Duve Institute, Avenue Hippocrate 75, 1200 Brussels, Belgium.

${ }^{9}$ Institute of Pharmaceutical Science, University of Veterinary and Animal Science Lahore, Pakistan.

${ }^{10}$ Center of Health Outcomes Research and Therapeutic Safety (Cohorts), School of Pharmaceutical Sciences, University of Phayao, Phayao, Thailand.

\begin{abstract}
Three-dimensional cell culture methods revolutionize the field of anticancer drug discovery, forming an important link-bridge between conventional in vitro and in vivo models and conferring significant clinical and biological relevant data. The current work presents an affordable yet reproducible method of generating homogenous 3D tumor spheroids. Also, a new open source software is adapted to perform an automated image analysis of 3D tumor spheroids and subsequently generate a list of morphological parameters of which could be utilized to determine the response of these spheroids toward treatments. Our data showed that this work could serve as a reliable 3D cell culture platform for preclinical cytotoxicity testing of natural products prior to the expensive and time-consuming animal models.
\end{abstract}

Keywords: Three-dimensional (3D); tumor spheroid; in vitro; curcumin

Received: $3^{\text {rd }}$ December 2018

Accepted: $10^{\text {th }}$ January 2019

Published Online: $18^{\text {th }}$ January 2019

*Correspondence to: Loh Teng Hern Tan, loh.tan@ monash.edu, Bey-Hing Goh, goh.bey.hing@monash. edu. School of Pharmacy, Monash University Malaysia, 47500 Bandar Sunway, Selangor Darul Ehsan, Malaysia.

Citation: Tan LTH, Low LE, Tang SY, et al. A reliable and affordable 3D tumor spheroid model for natural product drug discovery: a case study of curcumin. Prog Drug Discov Biomed Sci 2018; 1(1): a0000017

\section{Introduction}

Cancers are complex and heterogeneous pathological 'organ' that pose as life-threatening diseases which have inflicted over 18.1 million cancer related deaths globally in 2018 [1]. Despite the great effort shown in the post-genomics era by clinicians and researchers to optimize drug efficacy and reduce side effects, a steady decline is still evident in the success rate of new anticancer drugs entering early clinical development. This incident suggests that majority of pre-clinical models has yet to produce satisfactory results that can modify outcome of human cancer [2].
To date, majority of the pre-clinical in vitro models for cancer drug discovery largely rely on the testing the efficacy of therapeutics in cancer cell lines cultured in two dimensions (2D) which are generally highly reductionist, thereby these models do not incorporate the complexity arises from tumor heterogeneity and the microenvironment to which fail to capitulate in vivo-like growth of human solid tumors. In recent years, increasing interest in three-dimensional (3D) cell models has been evidenced, because the $3 \mathrm{D}$ cell models mimic more closely the native features of tumors in vivo as compared to the same cells grown in classical 2D culture flasks. When cells are grown in $3 \mathrm{D}$ environments, they express a number of unique physiological characteristics, including the 
development of more elaborate extracellular matrix and better intercellular communication [3-5]. Therefore, the in vitro $3 \mathrm{D}$-models have been proposed as the missing link that could offer the systems biology as an approach to bridge information from in silico, molecular target and $2 \mathrm{D}$-cell based screening, with in vivo models to enhance the predictability of cancer drug discovery $[6,7]$.

Natural products have contributed significantly to mankind for their wide variety of biological uses, particularly in human medicine, agriculture and industries [8]. Natural products are bioactive compounds originating from various natural sources, including plants [9-11], animals and microorganisms [12-15]. Among the different sources of natural products, plants represent one of the important sources for bioactive products in anticancer drug discovery $[16,17]$. For instance, a plant derived natural product, curcumin, which is a polyphenol isolat- ed from turmeric (rhizome of Curcuma longa) has been well known for its promising anticancer properties [18, 19]. The underlying anticancer mechanisms of curcumin have been extensively elucidated [20, 21]; however, majority of these findings were reported from 2D-cell culture while limited data was demonstrated in 3D-cell culture.

Herein, we devised and optimized a protocol of tumor spheroid culture generation for cytotoxicity testing of natural products such as curcumin was selected as the test agent in this study. Furthermore, the present work also highlighted a simple yet affordable approach in generating tumor spheroids based on hanging drop and agaroseoverlay method and the use of a user-friendly image-based efficacy analysis to ensure data robustness and minimum bias before initiating expensive and time-consuming animal models (Figure 1).

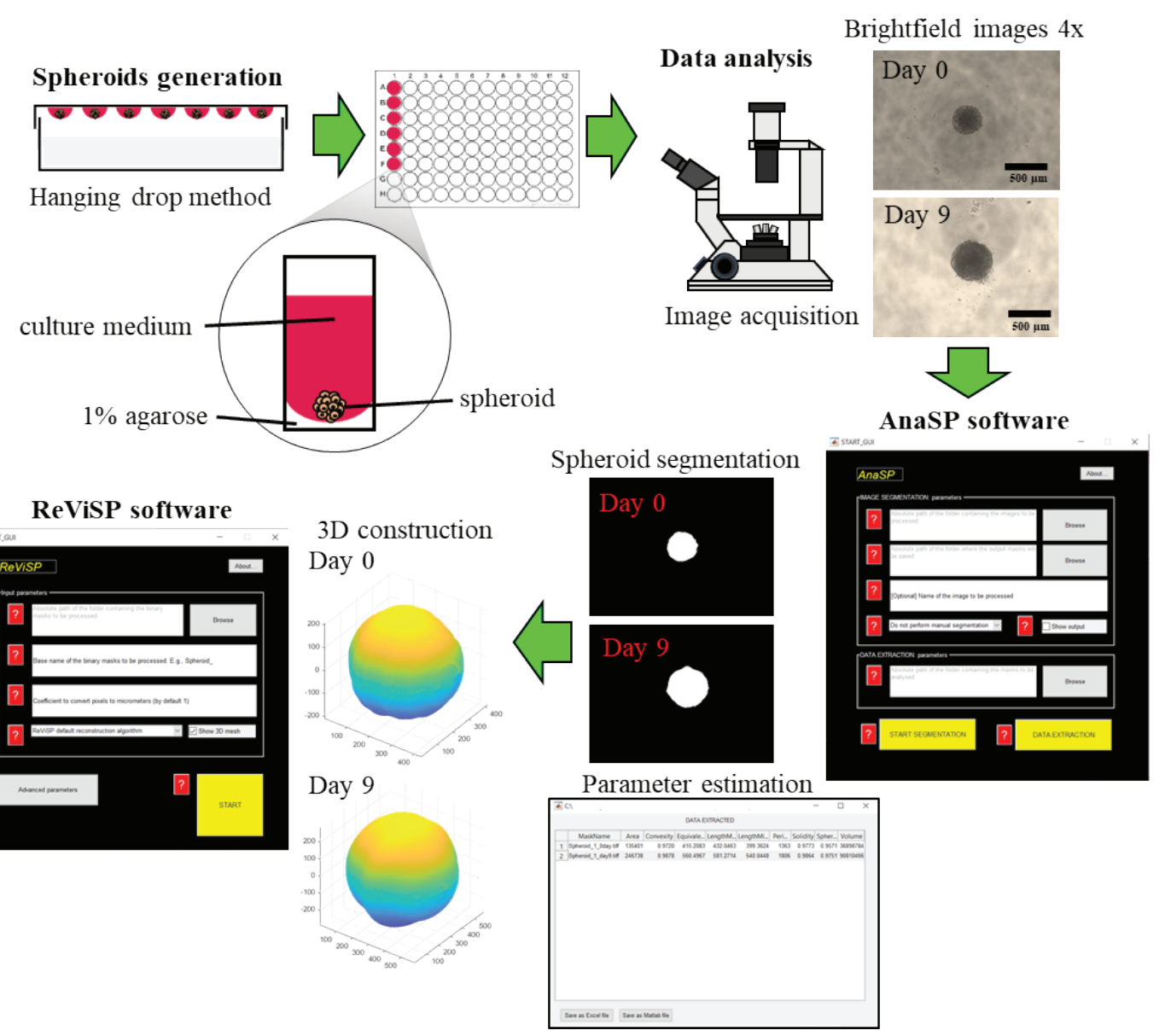

Figure 1. Schematic flow chart of the protocol of homogenous HCT116 spheroids generation and image-based data analysis.

\section{Method details}

Preparation and generation of spheroid cultures

\section{Materials}

- Cell culture facility equipped with $\mathrm{CO}_{2}$ incubator, biosafety cabinet, inverted bright-field microscope, microwave and centrifuge.

- $\quad$ Plastic consumables: cell culture flasks, sterile petri dishes, serological pipettes, $15 \mathrm{~mL}$ Falcon tubes, 96 well flat bottom plates, haemocytometer.
- Homo sapiens colon colorectal carcinoma (HCT116) cell line

- Cell culture medium: RPMI 1640 medium (Gibco) supplemented with $10 \% \mathrm{v} / \mathrm{v}$ of fetal bovine serum, $1 \%(\mathrm{v} / \mathrm{v})$ of 100x Antibiotic-Antimycotic (Gibco).

- $\quad$ Phosphate buffered saline $1 x$ (pH 7.4)

- $\quad$ Agarose powder

- $\quad$ TrypLE ${ }^{\mathrm{TM}}$ Select 1x (Gibco)

- Trypan blue stain $0.4 \%(\mathrm{w} / \mathrm{v})$ 
Procedure

HCT116 cells utilized for the experiments should be at logphase of growth, approximately $70-80 \%$ confluent.

(Note: Spheroid cultivation time, morphology can vary for different cell types. Therefore, initial cell seeding density should be pre-optimized to determine the optimal cell density for spheroid formation)

1. Remove used medium from cell culture. Rinse the cells with pre-warmed $\operatorname{PBS}\left(37^{\circ} \mathrm{C}\right)$.

2. Detach cells using $5 \mathrm{~mL}$ of $0.25 \mathrm{x}$ of TrypLE ${ }^{\mathrm{TM}}$ Select solution (Gibco). Incubate for at least 5 minutes at $37^{\circ} \mathrm{C}$, allowing complete detachment and dissociation of cells into single cell suspension.

3. Neutralise the detached cell suspension with $5 \mathrm{~mL}$ of cell culture medium.

4. Collect the cell pellet by transferring the suspension into $15 \mathrm{~mL}$ Falcon tube and centrifuge for 5 minutes at $1000 \mathrm{rpm}$

5. Resuspend the cell pellet in appropriate $(3-5 \mathrm{~mL})$ of fresh cell culture medium.

6. Aspirate a small volume of cell suspension for cell counting. Mix $50 \mu \mathrm{L}$ of cell suspension with $450 \mu \mathrm{L}$ Trypan blue and transfer $10 \mu \mathrm{L}$ into the haemocytometer.

7. Measure the cell number and adjust the cell density to approximately 100,000 cells $/ \mathrm{mL}$.

8. Place the drops of cell suspensions $(20 \mu \mathrm{L})$ with adjusted density on the lid of petri dishes. Gently invert the lid over the dishes containing $10 \mathrm{~mL}$ of sterile phosphate buffer saline to prevent evaporation.

9. Allow the sedimentation of the cells in the droplets by incubating the dishes in $5 \% \mathrm{CO}_{2}$ incubator at $37^{\circ} \mathrm{C}$ for 24 hours.

10. Harvest the cellular aggregates on the lid by using a pipette and transfer each cellular aggregate into agarose-coated 96 well plate $(50 \mu \mathrm{L}$ of $1 \%(\mathrm{w} / \mathrm{v})$ agarose in sterile $1 \times \mathrm{PBS})$.

10.1. Preparation of agarose-coated 96 well plate dissolve $1 \%(\mathrm{w} / \mathrm{v})$ agarose powder in $1 \mathrm{xPBS}$, facilitate the total dissolution of agarose by heating the mixture with microwave.

10.2. Adjust the pipette to $10 \mu \mathrm{L}$ dispensing volume, aspirate carefully the cellular aggregate from the droplets on the lids.

10.3. Dispense the $10 \mu \mathrm{L}$ culture medium containing the cellular aggregate into the agarose-coated 96 well plate pre-loaded with fresh $170 \mu \mathrm{L}$ culture medium.

11. Allow the cellular aggregates to undergo spheroidization (becoming spherical shape) by incubating in 5\% $\mathrm{CO}_{2}$ incubator at $37^{\circ} \mathrm{C}$ for another 24 hours.

\section{Cytotoxicity testing of natural product (curcumin)}

To validate the HCT116 3D-spheroid model for natural product drug discovery, the dose-dependent cytotoxic effect of curcumin on HCT116 spheroids is conducted.

Materials

- Cell culture facility equipped with $\mathrm{CO}_{2}$ incubator, biosafety cabinet, inverted bright-field microscope, imaging and data analysis software.

- Plastic consumables: serological pipettes, 96 well flat bottom plates, microcentrifuge tubes.

- Cell culture medium: RPMI 1640 medium (Gibco) supplemented with $10 \% \mathrm{v} / \mathrm{v}$ of fetal bovine serum, $1 \% \mathrm{v} / \mathrm{v}$ of $100 \mathrm{x}$ Antibiotic-Antimycotic (Gibco).

- Curcumin stock solution $(25 \mathrm{mg} / \mathrm{mL})$ in DMSO

Procedure

1. Measure the volume and sphericity of each spheroid before conducting the cytotoxicity experiment, ensuring uniform volume and sphericity between spheroid generated to improve the reproducibility of the experiment. Select spheroid with almost similar volume $[\mathrm{CV}]$ of $5 \%$ to $10 \%$. And exclude the spheroid with low sphericity index $($ SI $<0.9)$.

1.1. Acquire a brightfield image of each spheroid using inverted microscope.

1.2. Use AnaSP software (http://sourceforge. net/p/anasp/) to automatically compute the morphological parameters of each spheroid in term of its volume and sphericity index (SI) [22].

1.3. Use ReViSP software (http://sourceforge. net/p/revisp/) to construct the 3D structure of each spheroid [23].

2. Perform the cytotoxicity screening of the test agent on the spheroids by adding $20 \mu \mathrm{L}$ of a serial concentration of curcumin at a ten-fold higher of the desirable/test concentration.

3. Monitor the spheroid growth after respective cultivation time and acquire the brightfield images of the spheroids exposed to the test agent.

\section{Method validation}

\section{$\underline{\text { Spheroid culture generation }}$}

To evaluate the spheroid uniformity in term of the sphericity and volume generated by this protocol, the morphological parameters of each spheroid was determined. Our data showed that this protocol generated a set of homogenous spheroids with a narrow spheroid volume distribution of $0.0217 \pm 0.0015 \mathrm{~mm}^{3}(\mathrm{n}=\sim 60)$ (coefficient of variation $[\mathrm{CV}] 6 \%$ ). We also found that majority $(>98 \%)$ of the generated HCT116 spheroids acquired a spherical shape $(\mathrm{SI} \geq 0.90)$.

Cytotoxicity testing of curcumin against HCT116 spheroids 


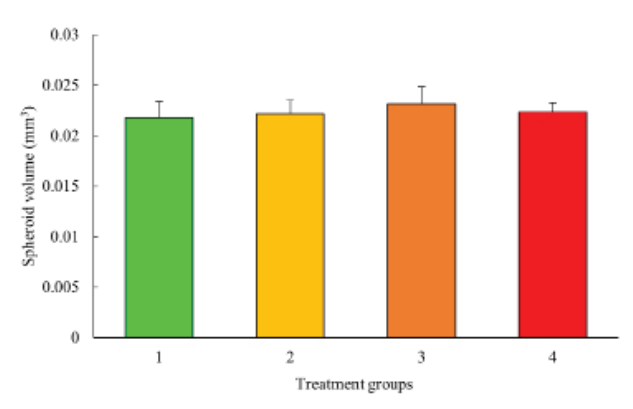

Figure 2. Uniformity of HCT116 spheroids $(n \geq 3)$ for each treatment group at an initial seeding density of 2000 cells/20uL at day 0 (before treatment).
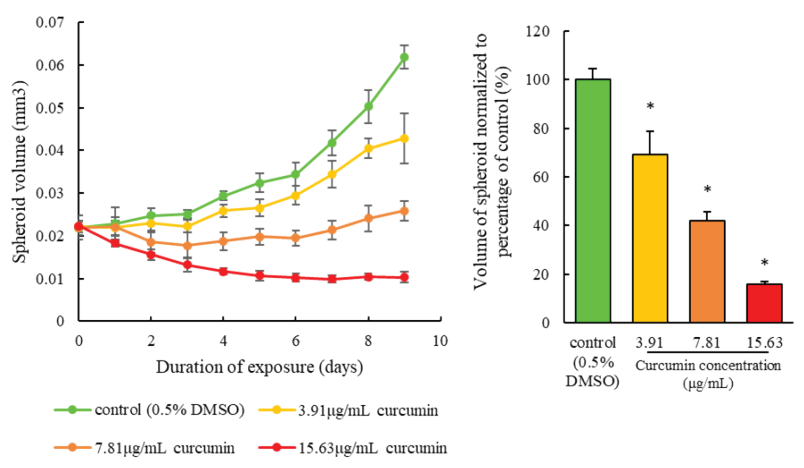

Figure 3. Evaluation of cytotoxicity effect of curcumin on the HCT116 spheroids $(n=4)$. a) The HCT116 spheroids were treated without (control) and with curcumin at $3.91,7.81$ and $15.63 \mu \mathrm{g} / \mathrm{mL}$ for 9 days. The efficacy of curcumin was evaluated based on the inhibition of HCT116 spheroids growth/size (volume) by measuring the estimated spheroid volume with the use of open source AnaSP and ReViSP software tools. Data points are expressed as mean values \pm SD. b) Bar graph shows the growth inhibition effect of curcumin on the HCT116 spheroids with significant reduction of spheroid volume after 9 days of exposure. Each bar represents the mean of the tumor volume normalized to percentage of control \pm SD.

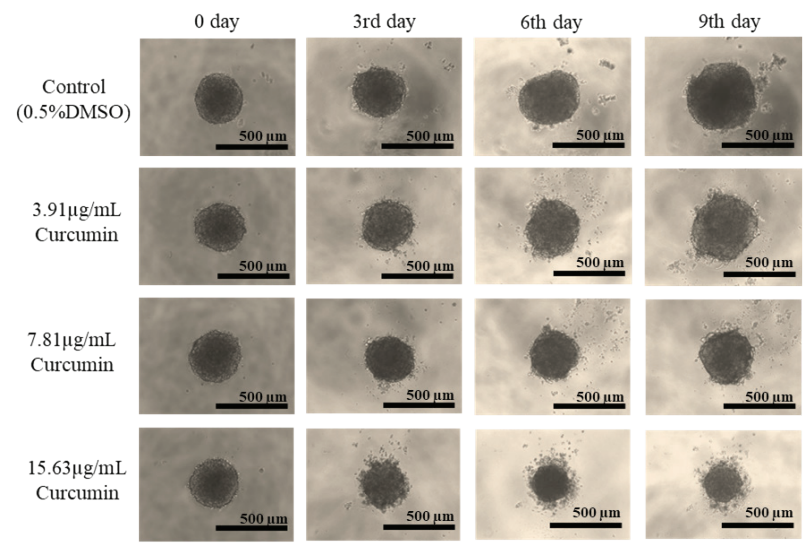

Figure 4. Brightfield images of HCT116 spheroids under treatment with and without curcumin for respective duration of exposure, day $\mathbf{0}$ (before treatment), 3,6 and 9. Scale bar at the bottom right of each image $=500 \mu \mathrm{m}$.

Based on Figure 3, the volume of the untreated HCT116 spheroids increased gradually from spheroidal volume of $0.022 \pm 0.0017 \mathrm{~mm}^{3}$ at day 0 to $0.059 \pm 0.0052 \mathrm{~mm}^{3}$ at day 9 (Figure 3). Upon exposure to curcumin, an evident reduction of the volume of the HCT116 spheroid was observed as the curcumin concentration increased (Figure $3 \mathrm{a} \& \mathrm{~b}$ ).
For instance, under the brightfield images showed that the curcumin treatment at $15.63 \mu \mathrm{g} / \mathrm{mL}$ resulted in a significant shrinkage of the HCT116 spheroids (Figure 4). In summary, the present work demonstrated an affordable and reliable platform for cytotoxicity testing of natural products in $3 \mathrm{D}$ cell culture system. On top of that, the imaging-based analysis employed in this platform offers a non-invasive quantification method for assessing the spheroids growth. Therefore, this platform allows easy recovery of the spheroids for further analysis. For instance, the post-exposed spheroids could be harvested via gentle aspiration and transfer to another appropriate well plate for further viability study. Taken together, the data and the established protocol of this study could serve a useful guide for future endeavours, especially to those new to the field of 3D cell culturebased drug discovery.

\section{Acknowledgement}

This research was financially supported by External Industry Grant (Biotek Abadi - Vote No. GBA-81811A) and MOSTI eScience funds (Project No. 02-02-10SF0215) awarded to B.-H.G.

Conflict of Interest The authors declared that there is no conflict of interest.

\section{References}

1. Bray F, Ferlay J, Soerjomataram I, et al., Global cancer statistics 2018: GLOBOCAN estimates of incidence and mortality worldwide for 36 cancers in 185 countries. CA: a cancer journal for clinicians, 2018. 68(6): 394-424.

2. Zanoni M, Piccinini F, Arienti C, et al., 3D tumor spheroid models for in vitro therapeutic screening: a systematic approach to enhance the biological relevance of data obtained. Scientific reports, 2016. 6: 19103-19103.

3. Daus AW, Goldhammer M, Layer PG, and Thielemann C, Electromagnetic exposure of scaffold-free three-dimensional cell culture systems. Bioelectromagnetics, 2011. 32(5): 351-9.

4. Lin RZ, Chou LF, Chien CC, and Chang HY, Dynamic analysis of hepatoma spheroid formation: roles of E-cadherin and beta1integrin. Cell Tissue Res, 2006. 324(3): 411-22.

5. Loessner D, Stok KS, Lutolf MP, et al., Bioengineered 3D platform to explore cell-ECM interactions and drug resistance of epithelial ovarian cancer cells. Biomaterials, 2010. 31(32): 8494-506.

6. Griffith LG and Swartz MA, Capturing complex 3D tissue physiology in vitro. Nat Rev Mol Cell Biol, 2006. 7(3): 211-24.

7. Pampaloni F, Reynaud EG, and Stelzer EH, The third dimension bridges the gap between cell culture and live tissue. Nat Rev Mol Cell Biol, 2007. 8(10): 839-45.

8. Newman DJ and Cragg GM, Natural products as sources of new drugs from 1981 to 2014. Journal of natural products, 2016. 79(3): 629-661.

9. Tan LTH, Lee LH, Yin WF, et al., Traditional Uses, Phytochemistry, and Bioactivities of Cananga odorata (Ylang-Ylang). EvidenceBased Complementary and Alternative Medicine, 2015. 2015: 30.

10. Ma DSL, Tan LT-H, Chan K-G, et al., Resveratrol-Potential Antibacterial Agent against Foodborne Pathogens. Frontiers in Pharmacology, 2018. 9: 102.

11. Wong DZ, Kadir HA, Lee CL, and Goh BH, Neuroprotective properties of Loranthus parasiticus aqueous fraction against oxidative stress-induced damage in NG108-15 cells. J Nat Med, 2012. 66(3): 544-51.

12. Tan LT-H, Ser H-L, Yin W-F, et al., Investigation of Antioxidative and Anticancer Potentials of Streptomyces sp. MUM256 Isolated from Malaysia Mangrove Soil. Frontiers in Microbiology, 2015. 6: 1316. 
13. Kemung HM, Tan LT-H, Khan TM, et al., Streptomyces as a Prominent Resource of Future Anti-MRSA Drugs. Frontiers in microbiology, 2018. 9: 2221

14. Tan LT-H, Chan K-G, Chan CK, et al., Antioxidative Potential of a Streptomyces sp. MUM292 Isolated from Mangrove Soil. BioMed Research International, 2018. 2018: 13 .

15. Ser H-L, Tan W-S, Cheng H-J, et al., Draft genome of starch-degrading actinobacterium, Microbacterium mangrovi MUSC 115T isolated from intertidal sediments. Progress in Drug Discovery \& Biomedical Science, 2018. 1(1)

16. Tang C, Hoo PC-X, Tan LT-H, et al., Golden Needle Mushroom: A Culinary Medicine with Evidenced-Based Biological Activities and Health Promoting Properties. Frontiers in Pharmacology, 2016. 7: 474.

17. Tan H-L, Chan K-G, Pusparajah P, Lee L-H, and Goh B-H, Gynura procumbens: An Overview of the Biological Activities. Frontiers in Pharmacology, 2016. 7: 52

18. Anand P, Sundaram C, Jhurani S, Kunnumakkara AB, and Aggarwal
BB, Curcumin and cancer: an "old-age" disease with an "age-old" solution Cancer letters, 2008. 267(1): 133-164.

19. Sa G and Das T, Anti cancer effects of curcumin: cycle of life and death. Cell division, 2008. 3(1): 1

20. Ravindran J, Prasad S, and Aggarwal BB, Curcumin and Cancer Cells: How Many Ways Can Curry Kill Tumor Cells Selectively? The AAPS Journal, 2009 11(3): 495-510.

21. Vallianou NG, Evangelopoulos A, Schizas N, and Kazazis C, Potential anticancer properties and mechanisms of action of curcumin Anticancer research, 2015. 35(2): 645-651.

22. Piccinini F, AnaSP. A software suite for automatic image analysis of multicellular spheroids. Computer Methods and Programs in Biomedicine, 2015. 119(1): 43-52.

23. Piccinini F, Tesei A, Arienti C, and Bevilacqua A, Cancer multicellular spheroids: volume assessment from a single $2 \mathrm{D}$ projection. Computer methods and programs in biomedicine, 2015. 118(2): 95-106. 